\title{
500 KILOVOLT CATHODE RAYS
}

\author{
By R. E. Vollrath* \\ California Institute of Technology
}

(Received June 2, 1931)

\begin{abstract}
The high potential $x$-ray tube at the California Institute has been modified so as to permit either x-rays or cathode rays to be produced. Electron currents up to 35 microamperes have been obtained through an aluminum window. A typical magnetic velocity spectrum of the high speed electrons passing out of an aluminum window is presented. The high velocity limit corresponds to about 500 kilovolts when the transformer voltage is 600 kilovolts peak. The velocity spectrum appears to be continuous and no absorption anomalies due to aluminum and lead foil are evident. The time required to obtain a velocity spectrum was reduced to a few minutes by allowing the electrons after being magnetically deflected, to pass through a copper foil into the air. The outside of the foil is coated with calcium tungstate and the photographic plate is placed in contact with the tungstate. It is noted that part of the photographic action of the high speed electrons is due to fluorescence of the glass backing of the photographic plates.
\end{abstract}

\section{INTRODUCTION}

T WAS shown in a previous paper ${ }^{1}$ that it is possible to operate the high potential x-ray tube at the California Institute of Technology with a hot cathode at about $600 \mathrm{kv}$ alternating current. Electron currents of at least 3 milliamperes can be handled in this type of tube; and therefore it appeared feasible to obtain, through a Lenard window, cathode rays corresponding to the above voltage and of sufficient intensity to permit magnetic velocity spectra to be recorded photographically in short exposures. This paper is a report of some preliminary work on these cathode rays and involves (1) the measurement of the potential difference between the electrodes of the tube and (2) the velocity spectrum of the electrons.

The measurement of the maximum or peak potential difference between the electrodes is a matter of considerable difficulty at these high voltages; and it was thought most reliable to determine it by calculation from the high velocity limit of the magnetic velocity spectrum of the electrons. The intensity of the cathode rays obtained made it possible to obtain the velocity spectrum of high speed electrons which had passed through foils of different substances for the purpose of discovering possible absorption edges due to quantum jumps higher in energy than the $K$ x-ray limit.

\section{Apparatus}

In order to permit the tube to be used for the production of either $\mathrm{x}$-rays or cathode rays the new electrode system in Fig. 1 designed by Dr. Lauritsen

* National Research Fellow.

${ }^{1}$ Lauritsen and Cassen, Phys. Rev. 36, 988 (1930). 
was used. The remainder of the tube was unchanged so that only the electrodes will be shown in Fig. 1. The upper or high potential electrode was furnished with a tungsten target which could be bombarded to produce $\mathrm{x}$-rays by electrons coming from a hot filament in the lower or grounded electrode. The upper electrode also had mounted in it a tungsten filament which could be heated by means of a 6 volt storage battery on top of the tube. The current through this filament is controlled by a rheostat in the filament circuit oper-

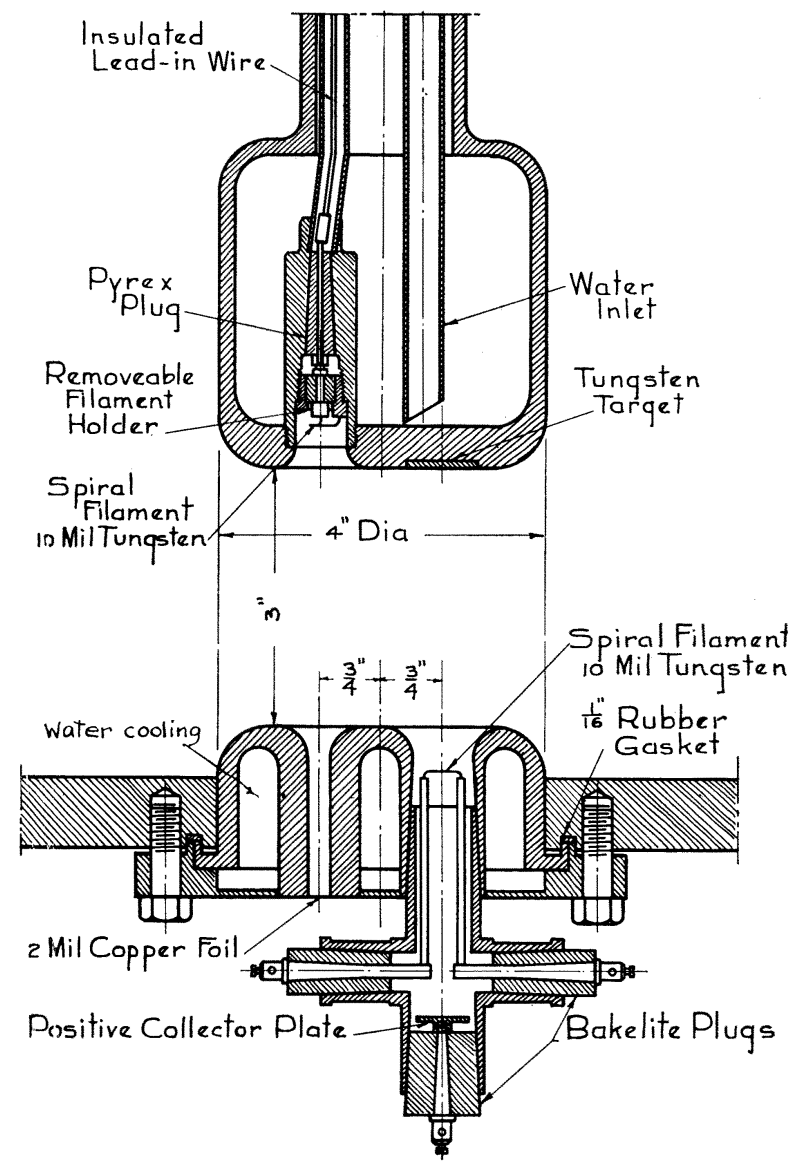

Fig. 1. Electrode system for producing either cathode rays or x-rays.

ated from the ground by means of long cords. Owing to the focussing effect of the recess in which the filament is mounted, electrons leave the upper electrode in a narrow beam which passes through the hole in the lower electrode and then through a thin copper window into the air.

At first mica and metal foils were fastened with sealing wax in the position shown in Fig. 1. However foils fastened in this manner were very easily punctured, probably due to deficient cooling. This difficulty was eliminated by using aluminum foil $0.004 \mathrm{~cm}$ thick clamped between the flanges of Fig. 2 . 
This arrangement was threaded in to the hole formerly closed off by the copper foil of Fig. 1. Electron currents up to 35 microamperes were obtained through this foil without damaging it. Whether this is anywhere near the maximum that can be sent through the foil cannot at present be stated because the

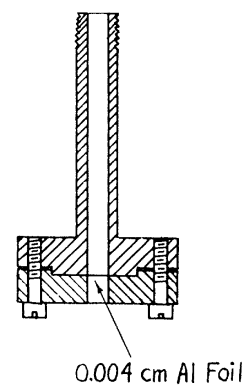

Fig. 2. Holder for aluminum window.

upper and lower electrodes were not sufficiently aligned and most of the electrons, amounting to 3 milliamperes, struck the walls of the hole. A few microamperes coming from the window in to the air could be seen as a cone of bluish luminescence extending almost a meter away from it.

\section{Velocity Spectrum}

In the velocity determination, the electrons entered an evacuated magnetic spectrograph through an aluminum foil $0.004 \mathrm{~cm}$ thick. The foil of the tube and that of the spectrograph were separated by a distance of $1 \mathrm{~cm}$, so that the velocity spectrum represents electrons which have passed through $0.008 \mathrm{~cm}$ of aluminum and $1 \mathrm{~cm}$ of air. Within the spectrograph a system of steel slits served to separate a beam of electrons $0.005 \mathrm{~cm}$ wide and $3 \mathrm{~mm}$ long

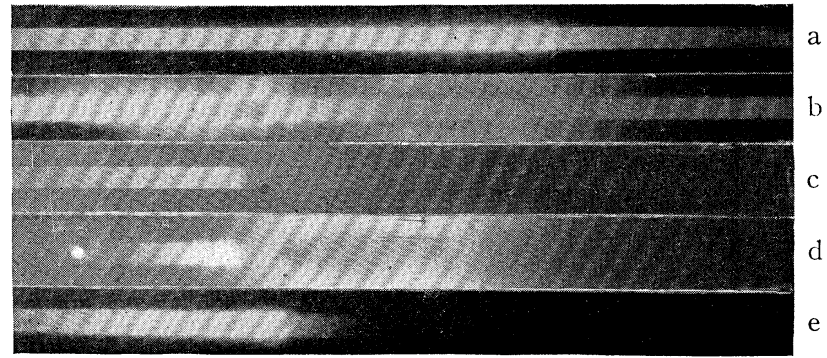

Fig. 3. Velocity spectra.

which was then bent into a semicircle by a magnetic field and fell on a photographic plate. The radius of curvature corresponding to a given position in the spectrum on the plate was determined from the position of a small spot photographed on the plate at a known distance from the slits. The magnetic field was measured after each exposure by means of a Grassot fluxmeter. The velocity was calculated from the measured radius of curvature of the electron 
beam and the magnetic field, making use of the relativistic mass of the electron. Spectrograms of sufficient intensity could be obtained in 20 minutes with 2 microamperes coming out of the aluminum window. Fig. 3a is a typical spectrum obtained in this way. The high velocity limit in this corresponds to $567 \mathrm{kv}$. The voltage at the transformer terminal during this run was $650 \mathrm{kv}$. The difference $83 \mathrm{kv}$ is most probably accounted for by a voltage drop in the water resistance in series with the tube and by a loss of velocity of the electrons passing through the foils. Spectrum $b$ shows the effect of overexposure resulting in the well known decrease of blackening when the plate is overexposed. Spectrum $c$ was obtained with the transformer voltage $630 \mathrm{kv}$. Here the high velocity limit was calculated to be $541 \mathrm{kv}$. Spectrum $d$ was obtained

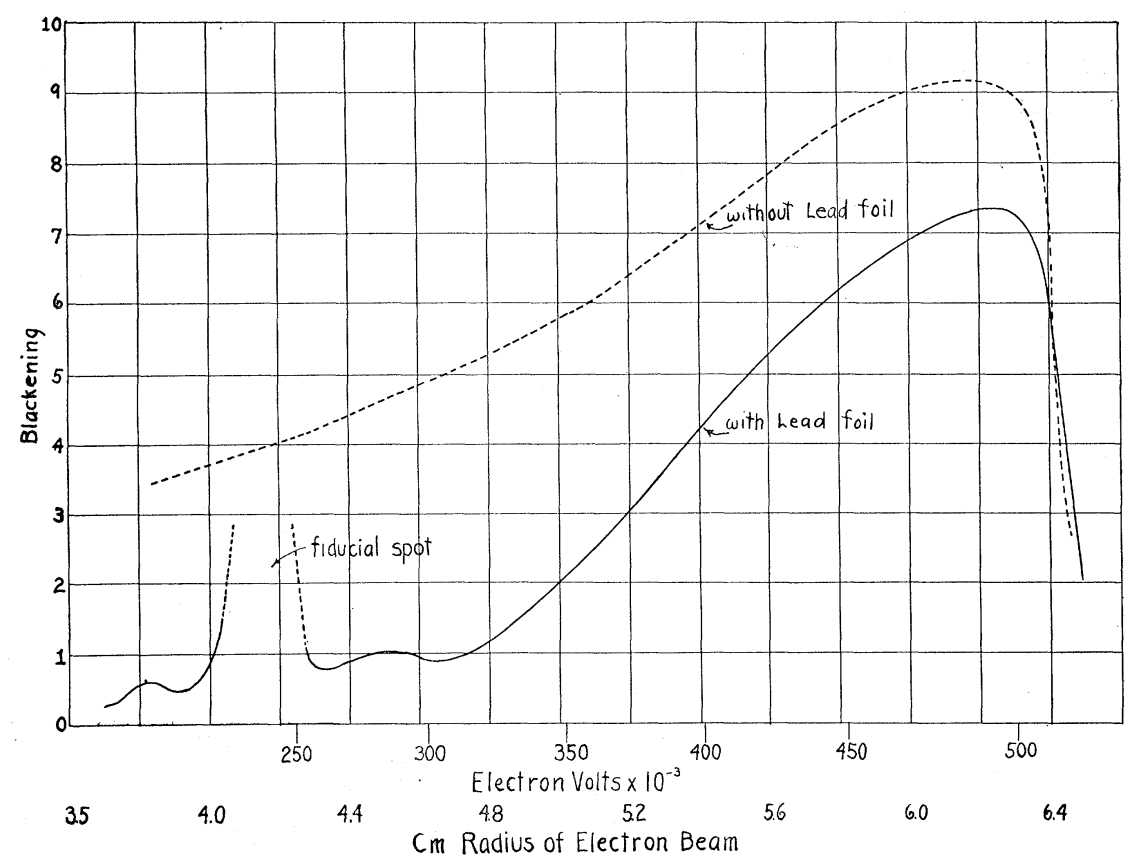

Fig. 4. Photometer record of electrons before and after passing through lead foil.

with the same voltage on the tube and the same magnetic field in the spectrograph, but the electrons were allowed to pass through a $0.003 \mathrm{~cm}$ lead foil before entering the spectrograph. A photometer record of spectrum $c$ and $d$ is shown in Fig. 4. Plate $d$ was exposed for 45 minutes while $c$ was exposed 15. The peculiar haze beyond the high velocity limit on $d$ is due to stray light entering an amber plug in the spectrograph. A close examination of the original negative showed no absorption discontinuities and this is verified by the photometer record. Similar plates were taken with copper and iron foils and here also no absorption anomalies were evident.

On most of the plates the spectrum was surrounded by a pronounced haze. Plate $b$ in Fig. 3 shows this most plainly. It was at first thought that this was due to $\mathrm{x}$-rays resulting from the impact of the electrons on the photo- 
graphic plate, and thus to serve as a means of investigating the efficiency of excitation of x-rays as a function of electron velocity. But the blackening was found to be due to visible fluorescence produced in the glass of the photographic plates by impact of electrons passing through the gelatine layer. A piece of black paper was placed over the photographic plate in the spectrograph and another plate placed on top of the paper with the sensitive gelatine layer in contact with the paper as in Fig. 5. After 20 minutes exposure the upper plate showed no blackening. But when the black paper was omitted the upper plate showed a blackening which was of the same order of intensity as the blackening produced by the electrons in the lower plate. Apparently a considerable fraction of the blackening in the electron spectrograms is due to this fluorescence. It is of course well known that glass fluoresces under the influence of electron bombardment. This fluorescence must be considered in any work on the blackening of photographic layers by high speed electrons.

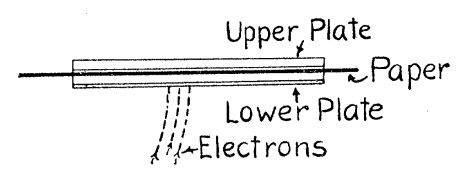

Fig. 5. Absorption of fluorescence by black paper.

With such high velocity electrons it was found feasible to obtain a velocity spectrum on a photographic plate outside of the spectrograph very much as oscillograms are obtained in the recently developed cathode ray oscillographs equipped with thin windows. The electrons after having been magnetically deflected were allowed to pass through a copper foil stretched over the gap between the semicircular pole faces of the electromagnet and to fall on a photographic plate placed with its sensitive layer in contact with the foil. A further improvement consisted in coating the outer surface of the copper foil with a thin film of collodion having suspended in it finely divided calcium tungstate. Electrons coming through the foil excited the tungstate to luminescence, and the velocity spectrum could be observed visually in a darkened room. Plate $e$ was obtained in 5 minutes when placed in contact with the tungstate layer while 2 microamperes were emitted from the window of the tube.

The writer wishes to thank Professor Millikan for placing the facilities of the laboratory at his disposal. 


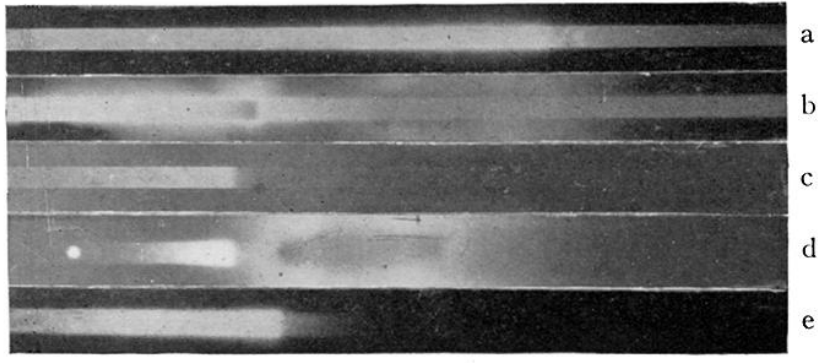

Fig. 3. Velocity spectra. 